\title{
Bioremediación de suelos contaminados con hidrocarburos derivados del petróleo
}

\author{
Joaquín Benavides López de Mesa Msc ${ }^{1}$, Gladis Quintero Msc ${ }^{1}$, Andrea Liliana Guevara Vizcaíno², Diana Carolina Jaimes \\ Cáceres², Sandra Milena Gutiérrez Riaño², Johanna Miranda García². \\ ${ }^{1}$ Docente- Investigador, Programa de Ciencias Básicas. Universidad de La Salle. \\ ${ }^{2}$ Facultad de Ciencias de la Salud, Universidad Colegio Mayor de Cundinamarca.
}

Correspondencia: jbenavides@jupiter.lasalle.edu.co

Recibido: 10-10-2005 / Aceptado: 22-06-2006

\begin{abstract}
Resumen
El manejo inadecuado de los materiales y residuos peligrosos ha generado a escala mundial, un problema de contaminación de suelos, aire y agua. Entre las más severas contaminaciones se destacan las que se produjeron y todavía se producen a causa de la extracción y el manejo del petróleo en todos los países productores de hidrocarburos. En nuestro país, el transporte de crudo y sus derivados se ha visto afectado considerablemente durante los últimos 18 años, por una permanente actividad terrorista contra los oleoductos e instalaciones petroleras. En el suelo los hidrocarburos impiden el intercambio gaseoso con la atmósfera, iniciando una serie de procesos físico-químicos simultáneos como evaporación y penetración, que dependiendo del tipo de hidrocarburo, temperatura, humedad, textura del suelo y cantidad vertida puede ser mas o menos lentos, ocasionando una mayor toxicidad, además de tener una moderada, alta o extrema salinidad, dificultando su tratamiento. Altos gradientes de salinidad pueden destruir la estructura terciaria de las proteínas, desnaturalizar enzimas y deshidratar células, lo cual es letal para muchos microorganismos usados para el tratamiento de aguas y suelos contaminados. En la presente revisión se analiza la biorremediacion como una alternativa "saludable" frente al deterioro progresivo de la calidad del medio ambiente por el derramamiento de crudos, ya que la esta problemática genera una amenaza real a la salud publica, así como la extinción de gran cantidad de especies vegetales y animales.
\end{abstract}

\section{Introducción}

La contaminación generada por el derramamiento de petróleo en Colombia; un problema alarmante.

En Colombia, el transporte de crudo y sus derivados se ha visto afectado considerablemente durante los últimos 18 años por una permanente actividad terrorista contra los oleoductos e instalaciones petroleras. Entre los años 1986 y 1998 las incursiones violentas de los grupos al margen de la ley ocasionaron el derramamiento de cerca de dos millones de barriles de petróleo, 7.6 veces el petróleo que se derramo en el desastre del buque Exxon Valdés entre Alaska y Canadá el 24 de Marzo de 1989, sobre ciénagas, pantanos, ríos, quebradas y suelos en su mayoría con vocación agrícola, pecuaria y pesquera; hasta dicha fecha el estimativo de las áreas afectadas fue de 6000 hectáreas de terrenos con potencial agrícola y pecuario, 2600 kilómetros de ríos y quebradas y 1600 hectáreas de ciénagas y humedales, 
originando grandes impactos negativos de carácter económico social y ambiental $(1,2)$.

Hasta noviembre de 1998 en Colombia se presentaron 920 ataques contra la infraestructura petrolera, 575 de ellos en el oleoducto Caño limón - Coveñas, que mediante roturas y abolladuras han perjudicado no solo a los ecosistemas y fuentes de producción y abastecimiento de las comunidades aledañas al oleoducto, sino a regiones por donde este transita. Las áreas perjudicadas por los derrames de petróleo se ubican principalmente en la zona alta de la llanura Araucana, en la región de la cuenca del rió Catatumbo, en la llanura del valle medio y medio bajo del río Magdalena; departamentos de Santander, Cesar y Sucre, principalmente, y en los departamentos del Putumayo y Nariño (2).

Entre los años 2000 y 2003 los ataques terroristas disminuyeron considerablemente con respecto a los años anteriores. En el año 2001, los grupos al margen de la ley realizaron 263 ataques, para el año 2002 la cifra llegó a 74 incursiones y para el primer semestre del 2003 la cantidad llegó a 60. Aunque los ataques han disminuido, los impactos ambientales permanecen en los diferentes ecosistemas afectados y se ven representados en las consecuencias ambientales.

\section{Consecuencias ambientales del derramamiento de crudo}

En el caso del suelo, los hidrocarburos impiden el intercambio gaseoso con la atmósfera, iniciando una serie de procesos físico-químicos simultáneos, como evaporación y penetración, que dependiendo del tipo de hidrocarburo, temperatura, humedad, textura del suelo y cantidad vertida pueden ser procesos mas o menos lentos lo que ocasiona una mayor toxicidad. Además de tener una moderada, alta o extrema salinidad, lo que dificulta su tratamiento, debido a que altos gradientes de salinidad pueden destruir la estructura terciaria de las proteínas, desnaturalizar enzimas y deshidratar células, lo cual es letal para muchos microorganismos usados para el tratamiento de aguas y suelos contaminados (2-4).
En el agua, al llegar a esta, la mancha generada por los hidrocarburos vertidos flota por diferencia de densidades impidiendo la entrada de luz y el intercambio gaseoso, dando comienzo a la solubilización de compuestos hidrosolubles y a la afección de diferentes poblaciones: la primera población afectada por un derrame es el plancton, en segundo lugar son afectados los macroinvertebrados, y la ultima población afectada son los Bentos o población de macroinvertebrados que viven en los fondos de los ríos y ciénagas (5).

Diferentes estudios han determinado el efecto de la contaminación con hidrocarburos en la germinación y crecimiento vegetativo de diferentes especies de pastos sometidos a diferentes concentraciones de hidrocarburo, concluyendo que hay una inhibición en la germinación del trébol común y un marcado retrasó en el crecimiento de todas las plantas evaluadas.

Así mismo, otros estudios evaluaron el efecto de los hidrocarburos poliaromáticos (PHA) en ecosistemas forestales y plantas madereras, demostrando un efecto de necrosis foliar (6) y reportando que aproximadamente 3200 hectáreas son afectadas por los derrames y el $90 \%$ de estos son pantanos o zonas inundables aledañas a plantas con tuberías corroídas por tener mas de 50 años de antigüedad, al igual que se emplean pozos sin crudo para almacenamiento de los cuáles un $30 \%$ están contaminados con desechos aceitosos (6).

Sin embargo, lo que complica la problemática actual de los sitios contaminados con hidrocarburos, es que hasta hace pocos años, prácticamente no existía una conciencia del grado de dificultad y del enorme costo de la remediación de suelos, cuerpos de agua y atmósfera contaminados, lo que representa hoy para la sociedad un gran costo económico (6). Dicha contaminación esta ocasionando el deterioro progresivo de la calidad del medio ambiente y genera una amenaza real a la salud publica, así como la extinción de gran cantidad de especies vegetales y animales.

www.unicolmayor 


\section{Principales fuentes de contaminación}

Una investigación realizada en 1981 por el instituto americano de petróleo (API) identifico entre las principales fuentes de contaminación (7):

Lodos de perforación de tipo inversa y recortes, estos lodos contienen un tipo de aceite muy similar a diesel en concentraciones de aproximadamente $10 \%$ y son sumamente arcillosos. Este material se deposita en presas, las cuales anteriormente eran construidas con materiales permeables y filtraban los hidrocarburos al medio ambiente.

Suelo contaminado por derrames de tuberías corroídas, existen campos petroleros con alrededor de cincuenta de 50 años de antigüedad, ubicados en zonas pantanosas, manglares u otras selvas inúndales. Los ductos de estos se instalaron conectando los pozos individuales a baterías de separación y desde ahí hasta las petroquímicas y refinería, generándose corrosión anaerobia, debido principalmente a bacterias reductoras de sulfato dando como resultado ductos corroídos y derramamientos. Los tipos de suelos afectados son de zonas bajas con altos contenidos de materia orgánica y arcilla y los menos afectados, son por lo general los más aptos para la agricultura por poseer texturas menos finas y alta fertilidad. Los sitios que se encuentren en la planicie costera, son los que más preocupan en caso de contaminación por el impacto que puede tener sobre los acuíferos, debido a su alta permeabilidad.

Tiraderos de desechos aceitosos semisólidos, se utilizan pozos que nunca produjeron petróleo o un pozo antiguo que no produce y esta tapado, puesto que nunca fueron diseñados para dicho fin y son construidos de materiales impermeables, muchas veces se termina el espacio disponible y se sigue depositando el relleno sobre la plataforma lo que resulta en escurrimientos e infiltraciones de hidrocarburos al medio ambiente cercano.
Sitios contaminados por descargas petroquímicas y refinerías, estos tienen sistemas antiguos de tratamiento de aguas residuales, las cuales generalmente contienen sales de los yacimientos de petróleo, lo que puede afectar los pantanos y cuerpos de agua.

\section{Biorremediación: una alternativa saludable}

Las prácticas de biorremediacion consisten en el uso de microorganismos como plantas, hongos, bacterias naturales o modificadas genéticamente para neutralizar sustancias toxicas, transformándolas en sustancias menos tóxicas o convirtiéndolas en inocuas para el ambiente y la salud humana $(6,8)$.

La biorremediación puede clasificarse de acuerdo al organismo que efectúe la degradación del compuesto xenobiótico. La fitorremediación, es el uso de plantas para la remoción de contaminantes de suelo y aguas, es una técnica apropiada para la remoción de metales pesados y radionucleos. Sin embargo, no se conoce sobre la habilidad de esta en el tratamiento de suelos con contaminantes orgánicos persistentes, un ejemplo lo constituye la especie Thlaspi caurulencens en suelos contaminados con zinc y cadmio donde se encontró que los eliminaba del suelo agrícola $(1,9)$

Así mismo, existen animales que actúan como agentes descontaminates, ya que pueden desarrollarse en medios con fuerte toxicidad y poseen en su interior microorganismo capaces de retener metales pesados; tal es el caso de la lombriz de tierra (lumbricus terrestris) la cuál absorbe los contaminantes a través de los tejidos y los acumula en las vías digestivas $(1,10)$.

Las bacterias son las más empleadas en el proceso de biorremediación, aunque también se han empleado otros microorganismos como hongos, algas, Cianobacterias y Actinomicetes para la degradación de compuestos tóxicos en el suelo (1). 
La biorremediación del suelo, puede ser dividida en estrategias in situ y ex situ. In situ corresponde a la biorremediación referente a tratamientos que no requieren excavación del suelo contaminado; ex situ es un método donde se excava el suelo o el material a tratar y se le maneja en un sistema controlado como una celda de landfarming o algún tipo de biorreactor (11-13).

Las técnicas de biorremediación in situ presentan una mayor ventaja sobre las ex situ por el menor costo y la disminución de la generación de residuos a eliminar en la superficie (14). La biorremediación in situ es la más aplicada y utiliza microorganismos autóctonos, estos en el suelo pueden degradar un gran número de constituyentes de lodo pero su eficacia y su población son afectados cuando algunos contaminantes tóxicos están presentes en altas concentraciones. La reintroducción de microorganismos aislados de un sitio contaminado ayuda a resolver este problema ya que los microorganismos pueden degradar los constituyentes y tiene una gran tolerancia a la toxicidad (15).

Los métodos, más empleados en biorremediación son:

(i) Biofiltración, en esta entra el aire contaminado a unos biorreactores en los cuales la flora microbiana degrada los contaminantes volátiles en dióxido de carbono, agua y biomasa, (ii) Bioventing, el cuál es un proceso de biorremediacion in situ que consiste en la ventilación forzada del suelo mediante la inyección de $\mathrm{O}_{2}$ en la zona no saturada mediante pozos de inyección; debido a la aireación se va a favorecer la degradación de los hidrocarburos por volatilización y migración de la fase más volátil del contaminante, y por biodegradación, ya que al incrementar la oxigenación del suelo se va a estimular la actividad microbiana, (iii) el Biosparging es un método in situ que combina el efecto de la ventilación con la utilización de los microorganismos autóctonos para degradar compuestos orgánicos absorbidos por el suelo en la zona saturada. En este el aire y los nutrientes se inyectan en la zona saturada para mejorar la actividad de los microorganismos presentes.
Está técnica se utiliza para la degradación de compuestos orgánicos en el suelo y aguas subterráneas. (iv) las biopilas, son un tipo de biorremediación de tipo ex situ en condiciones no saturadas, consiste en la reducción de la concentración de contaminantes derivados del petróleo de suelos excavados mediante el uso de la biodegradación. La técnica consiste en la formación de pilas de material biodegradable de dimensiones variables formadas por suelos contaminados y materia orgánica, compost, en esta se aplica una aireación activa volteando la pila o pasiva por tubos de aireación. Este principio se puede aplicar a la mayoría de los compuestos orgánicos siendo más eficaz en los ligeros (16-21).

Entre los factores que influyen en la aplicación de esta técnica se destacan: los hidrocarburos deben ser no halogenados con concentraciones en suelo menores a $50.000 \mathrm{ppm}$, superficie de trabajo relativamente grandes, necesidad de una población microbiana mayor a 1000UFC (Unidades Formadoras de Colonias) por gramo de suelo.

(v) la atenuación natural, es una técnica de biorremediación in situ de bajo costo, su característica principal es la utilización de los procesos físicoquímicos de interacción contaminante suelo y los proceso de biodegradación tienen lugar de forma natural. estos procesos se conocen como procesos de biotransformación natural, que van a reducir la concentración de los contaminates. Entre ellos se encuentra la dilución, la dispersión, volatilización, adsorción, biodegradación y la reacciones químicas que se producen en el suelo (22).

Las técnicas de recuperación para acuíferos contaminados más empleadas son el bombeo de agua contaminada y posterior tratamiento en superficie, inyección de soluciones acuosas con reactivos e inyección de airea para eliminar sustancias volátiles $y$ el uso de microorganismos para trasformar y degradar el contaminante. 


\section{Microorganismos degradadores de petróleo}

En Long Beach (California), aplicaron 1a biorremediación in situ en suelos contaminado con aceite diesel mediante el uso de microorganismos autóctonos complementada con la adición de nutrientes y oxigeno en el suelo bioestimulación (23), e inoculación de una mezcla enriquecida de consorcios bacterianos previamente extraída del mismo suelo bioaumentación. Esto permitió encontrar consorcios bacterianos degradadores de hidrocarburos identificados por secuenciación de genes 16S-RNA, demostrando la presencia de Bacillus cereus, Bacillus sphaericus, Bacillus fusiformis, Bacillus pumilis, Acinetobacter junii, y Pseudomonas sp (24).

El estudio hecho sobre la adaptación y selección de microorganismos autóctonos en medios de cultivo enriquecidos con petróleo crudo realizado por Maria del Carmen Rivera-Cruz 2002 indicó que los microorganismos con crecimiento abundante aislados de suelos receptores de derrames recientes y crónicos de petróleo crudo y otros no contaminados, son considerados degradadores de petróleo (25).

Los microorganismos aislados en suelos poseen actividades de peroxidasas y oxigenasas, que permiten la oxidación de algunas fracciones del petróleo (26). Esta oxidación cambia las propiedades de los compuestos haciendolos susceptibles a ataques secundarios y facilitando su conversión a bioxido de carbono y agua $(27,28)$.

Uno de los géneros bacterianos más explotados en bioprocesos no convencionales es Rhodococcus, un grupo único consistente en microorganismos que presentan una gran diversidad metabólica, capaz de transformar, biodegradar y utilizar como única fuente de carbono compuestos hidrófobos (29).

Los Rhodococcus son aeróbios, Gram positivos, inmóviles, Nocardiformes, Actinomicetos, que en algunas ocasiones presentan pequeñas proyecciones filamentosas. Las características bioquímicas encontradas en algunas cepas son la producción de poli-3hidroxialcanoatos, acumulación de metales pesados y enzimas útiles como la fenilalanina, deshidrogenasa y endoglucosidasas.

El Rhododoccus poseen una gran variedad de vías metabólicas para la degradación y modificación de compuestos aromáticos, incluyendo las actividades de di-oxigenasa y mono-oxigenasa sobre anillos, así como la actividad de ruptura de catecol. Algunas cepas presentan también la vía del 3-oxoadipato. Lo anterior sumado a su capacidad de crecimiento en medios con escasos nutrientes, la carencia de un sistema de represión catabólica y su persistencia ambiental las hacen excelentes candidatas para los tratamientos de biorremediación (29).

El Rhodococcus sp. utiliza el dibenzotiofeno (DBT) como única fuente de azufre, el DBT y sus derivados son los órganoazufrados más abundantes en el diesel primario. En México, investigadores del Instituto Mexicano del Petróleo han aislado cepas de Rhodococcus de sitios contaminados con petróleo capaces de desulfurar muestras de diesel. Otros microorganismos reportados como capaces de utilizar el DBT como fuente de azufre son las cepas de Gordona y Nocardia sp. Dentro de las aplicaciones industriales y ambientales, se incluye la producción de ácido acrílico y acrilamida, conversión de esteroides, biorremediacion de hidrocarburos clorados y fenoles, a lo que se añade su gran capacidad de degradar hidrocarburos alifaticos halogenados y numerosos compuestos aromaticos, como los PHA's (hidrocarburos policiclicos aromáticos) (30), evidenciandose que tanto $R$. rhodochorus como $R$. erythropolis demostraron ser una excepción, pues la degradación de naftaleno por parte de estos no es significativa, debido a que la actividad degradadora de PAHs por parte de estos microorganismos, se ve regulada por las proteobacterias del medio afectado (31-33).

Otras aplicaciones potenciales son la producción de biosurfactantes emulsificantes naturales de hidrocarburos, los cuales son producidos por algunos hongos o bacterias y el análisis de su metabolismo permite obtener biomoléculas a escala comercial y la biodesulfuración de combustibles, la deshalogenación 
de emisiones gaseosas y la construcción de biosensores (34).

Nuevas especies de Rhodococcus que presentan una nueva mutación tienen la capacidad de degradar compuestos alifáticos con enlaces dobles principalmente en el noveno carbono del grupo metilo terminal (alquenos). Las bacterias del género Pseudomonas poseen la habilidad para utilizar diversos substratos, incluyendo aquellos creados por el petróleo. Las Pseudomonas son bacterias Gram negativas, obicuas, que pertenecen a la subclase gamma de las Proteobacterias.

Las Pseudomonas son bacterias productoras de biosurfactantes como los ramnolipidos involucrados en procesos de remoción de aceites y productos relacionados, Bushnell y Hass fueron de los primeros en describir bacterias productoras de biosurfactantes, como el Corynebacterium simplex y cepas de Pseudomonas $(29,35)$.

Algunos microorganismos productores de biosurfactantes extracelulares que solubilizan y facilitan la penetración de los hidrocarburos a través de la pared celular hidrofílica; contienen además enzimas degradadoras de hidrocarburos en la membrana citoplasmática. La Pseudomonas aeruginosa, es otro de los microorganismos más usado y estudiado en biorremediación y presenta una serie de actividades naturales sobre xenobióticos. Lamentablemente, también es conocida por ser un patógeno oportunista en humanos y causante de complicaciones graves en personas inmunosuprimidas, con quemaduras severas o con fibrosis quística. Por estas razones existe mucho interés en el estudio de las relaciones filogenéticas entre serotipos clínicos y ambientales (36).

Estudios con relación al desempeño metabólico de la Pseudomonas aeruginosa ha permitido identificarla como degradadota de gran cantidad de sustratos como el n-hexadecano, mineralización de compuestos alifáticos en condiciones anaerobias, y degradadora de hidrocarburos aromáticos y poli aromáticos, así como del pireno en estudios in vitro $(37,38)$.
La $P$. aeruginosa tiene la capacidad de sintetizar ramnolipidos cuando se encuentra en la fase estacionaria de su crecimiento, por tal razón esto sólo se puede realizar en la primera fase del proceso de biorremediacion y contribuyendo así con la movilización y solubilización de los contaminantes durante la fase siguiente de mineralización. Al mismo tiempo que pueden tranformarse bajo microcosmos en el suelo con un tratamiento físico o químico especifico, característica que comparte con el Agrobacterium tumefasciens (39).

La Pseudomona putida es un saprofito del suelo, oportunista, cosmopolita, metabólicamente versátil, por poseer una dioxigenasa inicial, una tolueno dioxigenasa, aunque no presenta la dioxigenasa específica para los PAHs por lo cuál es una buena candidata para las aplicaciones biotecnológicas, tales como agricultura, biocatálisis, biorremediación, biocontrol en protección de las plantas y producción de bioplásticos (14).

La $P$. putida posee la capacidad de colonizar la rizosfera de plantas de cosecha y una gran capacidad metabólica que facilita el desarrollo de biopesticidas y promotores de crecimiento de la planta. La degradación de los alcanos por Pseudomona putida se ha estudiado por secuenciación en el plasmido OCT que codifica una enzima dioxigenasa que convierte alcanos a aldehídos a través del hidroperoxidasa del n-alkyl sin un intermediario del alcohol, conocido como la vía de Finnerty; un proceso similar lo presentan los géneros Acinetobacter $s p$ y Nocardiodes $s p$. aunque ellos no poseen este plasmido.

La Pseudomonas fluorescens es degradadora de naftaleno y fenantreno, ventaja que tiene frente a las otras Pseudomonas, que solo metabolizan naftaleno $\mathrm{y}$ asfaltenos (36).

Estudios realizados demuestran que Flavobacterium y Pseudomonas son los microorganismos más aislados en la fase de degradación de los TPH (Hidrocarburos Totales). La Pseudomonas stutzeri es una degradadora de PHA's (33). 
Burkholderia es otro género bacteriano utilizado para biorremediación de herbicidas y pesticidas recalcitrantes y también es usado para proteger cultivos contra hongos. Debido a su genoma extremadamente flexible, Burkholderia cepacia, bacilo Gram negativo no fermentador, productora de pigmento amarillo tiene una gran capacidad mutagenica y adaptativa, ha sido recuperada a partir de agua y superficies húmedas, es resistente a múltiples antibióticos y ésta capacidad es altamente transmisible entre especies. Por todas estas razones, la selección de cepas "seguras" para su uso ambiental no es posible por el momento y su uso en la agricultura también debe ser cauteloso. Aunque es una excelente degradadora de los hidrocarburos aromáticos $(34,40)$

El Acinetobacter sp es un bacilo Gram negativo, es productor de ácido a partir de la glucosa, se desarrolla a 41 y $44^{\circ} \mathrm{C}$, produce $\alpha$-xilosa y utiliza el malato. Dentro de las especies de importancia ambiental se destacan, A. baumanii, Acinetobacter calcoaceticus carente de ácido metílico. Las cepas de Acinetobacter baumanii son eficientes en la degradación de fracciones de alcanos (41).

Los productos de petróleo ampliamente usados como gasolina, keroseno y Diesel son contaminantes comunes del ambiente, se ha observado que la biodegradación de gasolina por microflora de suelo y agua de sitios contaminados es eficiente; compuestos como el benceno, tolueno, etilbenceno, y n-alcanos son realmente biodegradables. Se aisló una cepa con una alta capacidad de degradar dichos compuestos identificada como Mycobacterium austroafricanum (42).

Se selecciono una cepa en un acuífero contaminado con gasolina, la cuál tiene la capacidad de utilizar el isoctano (2,2,4-trimetilpentano) como única fuente de carbono y energía. El microorganismo aislado fue identificado por medio de la secuenciación del $16 \mathrm{~S}$ ribosomal. Se identifico una cepa perteneciente a Mycobacterium austroafricanum(42); y otra cepa como Mycobacterium sp. Esta de manera inusual utilizo como fuente de carbono y sustrato de crecimiento n-alcanos y multimetil, donde degrado el metil t-butil eter, y grupos 3-metil, posiblemente por mecanismos de carboxilación y deacetilación; logrando finalmente la degradación del $86 \%$ de los sustratos contenidos en este sitio contaminado con gasolina (43).

La degradación de gasolina por microflora de suelo y agua en sitios contaminados, es realmente eficiente, se puede alcanzar un nivel de biodegradación hasta del 90\%. La limitación de esta microflora aparentemente se presenta a nivel de la degradación de compuestos como el ciclohexano y trimetilpentano, los cuales se presentan como compuestos recalcitrantes (44).

La microflora degradadora de ciclohexano e isoctano es seleccionada directamente de las muestras tomadas del ambiente. La cepa que utiliza el ciclohexano como única fuente de carbono no se ha logrado aislar pura, debido a los fenómenos de metabolismo y cometabolismo involucrados en la degradación de este compuesto.

En cuanto a la capacidad de degradación del isoctano, resulta ser complicada en las muestras naturales, solo se ha aislado el Mycobacterium austroafricanum con esta capacidad, así como también se ha encontrado que esta cepa se caracteriza por el uso de ciclohexano como un cosustrato, pues su habilidad radica en el uso de isoctano como única fuente de carbono (42).

Otro género estudiado dentro de las técnicas de biorremediacion es Sphingomonas, bacilos no fermentadores y dentro de estos la Sphingomona wittichi es un microorganismo capaz de degradar en condiciones anaerobias el 2.7diclorobenceno, produciendo el metabolito 4 clorocatenol y el 1,2,3,4 tetraclorodibenceno, y cepas como Sphingomonas yanoikuyae, y Sphingomonas paucimobilis como degradadoras de $\mathrm{PAH}^{\text {'s }}$ utilizandolos como unica fuente de energía, tienen actividad Catecol 2,3dioxigenasa, fenantreno y antraceno.

Se han aislado cepas capaces de metabolizar naftaleno y fenatreno como única fuente de carbono, 
estas fueron taxonómicamente implicadas en diferentes subclases de las Proteobacterias (Sphingomonas sp, Acidovorax sp, Comamonas sp, y Pseudomonas $s p)$, y a bacterias Gram positivas con bajo y alto contenido de DNA G+C (Paenibacillus sp y Rhodococcus sp, respectivamente) (45).

Alcaligenes, Micobacterium y Bacteroides han sido reportados como degradadores de hidrocarburos de petróleo, y estos filotipos son candidatos para el tratamiento de terrenos contaminados con TPH. Sin embargo su poca abundancia se convierte en una desventaja para su aplicación (46).

La degradación del bifenil policlorinato PCBs y otros hidrocarburos por microorganismos como Comamonas acidovorans, Acinetobacter calcoaceticus, Achromobacter sp. Pseudomonas sp. Flavobacterium devorans, Bacillus lentus, Bacillus mascerans y Bacillus thuringensis ha sido aplicada con buenos resultados para el tratamiento de los mismos $(47,48)$.

Se ha referenciado que las Comamonas son degradadoras de fenaltreno, Gordona $s p$. degrada el Pireno, de 5 a 35 días luego de ser inoculada. Azoarcus spp. puede degradar benceno, tolueno, etilbenzeno y xileno $(31,32)$.

En el estudio hecho por Christopher W. Kaplan y Christopher L. Kitts en cuanto al dinamismo de las comunidades bacterianas en relación con la concentración de petróleo en el suelo, la importancia de las comunidades microbianas radica en su maquinaria celular como responsable de la bioconversión de estos contaminantes (43).

Los microorganismos que conforman el consorcio microbiano extremo halótolerante para la biodegradación de crudos es altamente empleados en medios con gran cantidad de salinidad y contaminados con benceno.

Las poblaciones microbianas usadas para degradar hidrocarburos recalcitrantes (aromáticos), son menos eficientes, debido a que producen sustancias tóxicas que pueden inhibir los microorganismos degradadores de aceite diesel.
La persistencia de los PAHs en el ambiente se deben a factores como los nutrientes, la viabilidad, la temperatura, el oxigeno y la presencia de microorganismo degradadores y el mayor problema para la degradación de esto es la baja solubilidad en agua para poder implementar un sistema aeróbico, por esto la degradación anaeróbica a bajas temperaturas ha sido estudiada como posible causa de una baja degradación donde encontraron que las bajas temperaturas no afectan la población microbiana sino que la disponibilidad de oxigeno, siendo este el agente más limitante y que conducía a formación metabolitos tóxicos que inhibían el crecimiento bacteriano $(34,44,49,50)$.

\section{Referencias}

1. Atlas R, Bartha R. Ecología microbiana y microbiología ambiental. Ed. Addison Wesley. Madrid. 2002. 561p.

2. Restrepo R. Derrame de hidrocarburos. Impacto en los ecosistemas tropicales. ECOPETROL Instituto Colombiano de Petróleo. 2002.

3. Randy H. Schroeder A, Domínguez V, García L. Potencial de la biorremediación de suelo y agua impactados por petróleo en el trópico de México. Terra. 1999; 17(2): 159-174.

4. Siva S, Brett R, Tessa M, Vogeler I, Clothier B, Grant L. Northcott and Don McNaughton. Bioremediation of soils contaminated with organic compounds. 2004. En: http: // www.regional.org.ou/a u/ass si/supersoi $12004 / \mathrm{pdf} /$ 1455 sivakumarans.pdf.

5. Fatima B, Flavio A, Oliveira C, Benedict O, William T. Bioremediation of soil contaminated by diesel oil. Brazilian Journal Microbiology. 2003;34(1).

6. Schmidt W. Suelos contaminados con hidrocarburos: la biorremediación como una solución ecológicamente compatible. Cooperación Técnica Alemana (GTZ). 2000. En: w w w. g t z.org. mx/sitios - cont a m/a r t i c u los / biorremed_Mex2.pdf.

7. Benavides J , Roldan F, Ocampo A, Londoño M, Pardo A, Perdomo C. Efecto de la adición de nutrientes (nitrógeno y fósforo) e identificación de microorganismos degradadores de hidrocarburos de suelos contaminados con petróleo. (Fase I). Trabajo de grado (Ingeniera (s) ambiental). Universidad de la Salle. Facultad de Ingeniería ambiental. Ambiental. Bogotá, 2004.

8. Plaza G, Otero M, Torres N, Velásquez M, Corbalan E, Rodríguez T. Biorremediación en suelos contaminados con hidrocarburos. Avances en Energías Renovables y Medio Ambiente.2001;5: 163-167.

9. Blaz S, Imahne I, Gorazd A, James T. Nitroux oxide reductase (nosZ) gene fragments differ between native and cultivated Michigan soils. Applied and Environmental Microbiology.2004; 70(1): 301-309.

10. Rojas N, Rodríguez R, Enríquez F, Martínez J, Poggi H. Transformer oil degradation by an indigenous microflora isolated from a contaminated soil. Departamento de Biotecnología y Bioingeniería, Centro de Investigación y de Estudios avanzados.1999: 15-24.

11. Bongkeun S, Norberto J, Palleroni M, et al. Isolation and characterization of diverse halobenzoate - degrading denitrifying bacteria from soils and sediments. Applied and Environmental Microbiology. 2000; 66(8): 3446-3453. 
12. Cèbron A, Berthe T, Garnier J. Nitrification and Nitrifying Bacteria in the lower Seine river and estuary (France). Applied and Environmental Microbiology. 2003; 69(12): 7091-7100.

13. Ebru C. Isolation and characterization of aerobic denitrifiers from agricultural soil. 2004; 29: 9 -14.

14. Daane L, Harjono I, Zylstra G, Häggblom M. Isolation and characterization of polycyclic Aromatic hydrocarbondegrading bacteria associated with the Rhizosphere of salt Marsh plants. Applied and Environmental Microbiology.2001; 67(6):2683-2691

15. Dryidale G, Kasan H, Bux F. Denitrification bacteria during activated sludge treatment. Water SA. 1999; 25: 357-362.

16. Maroto A, Rogel J. Aplicación de sistemas de biorremediación de suelos y aguas contaminadas por hidrocarburos. Geocisa. División de protección ambiental de suelos. En: http:// www.igme.es/internet/web_aguas/igme/publica/ con_recu_acuiferos $/ 0.28$ pdf.

17. Bonilla A, Cuesta P, Hierro B. Recuperación de suelos contaminados por hidrocarburos. Utilización de la técnica de venteo y aplicación de modelos de simulación. 1999.

18. Gispert $\mathrm{M}$. Les pèrdues de $\mathrm{N}_{2} \mathrm{O}$ del sòl. institució catalana d'estudis agraris. 1999; 21-28.

19. Rojas N, Martínez J, Zermeño L, Rodríguez R. Levels of polychlorinated Biphenyls in Mexican Soils and Their Biodegradation using bioaugmentation. Environmental contamination and toxicology. 2003; 70:63-70.

20. Roane T, Josephson K, Pepper I. Dual-Bioaugmentation strategy to enhance remediation of contaminated soil. Applied and Environmental Microbiology.2000; 67(7): 3208-3215.

21. Ulla B, Flemming E, Carsten J. Method for spiking soil samples with organic compounds. Applied and Environmental Microbiology. 2002; 68(4): 1808-1816.

22. Lee H, Lee J, Cheon J, Lee K. Attenuation of Petroleum Hydrocarbons in Smear Zones: A Case Study. Journal of Environmental Engineering. 2001;127(7): 639-647.

23. Cunningham C, Philp J. Comparison of Bioaugmentation and Bioestimulation in ex situ treatment of Diesel Contaminated Soil. Land Contamination and Reclamation. 2000; 8 (4): 261269.

24. Téllez J, Valderrama B. Microbiología del petróleo y sus derivados. Instituto de Biotecnología, Universidad Nacional Autónoma de México. 2000.

25. Rivera M, Ferrera R, Volke V, Rodríguez R, Linares L. Adaptación y Selección de Microorganismos Autóctonos en Medios de Cultivos Enriquecidos con Petróleo Crudo. Terra. 2000; 20: 423-434.

26. Rich J, Heichen R, Bottomley P, Cromack J, Myrold D. Community composition and functioning od denitrifying bacteria from adjacent meadow and forest soil. Applied and Environmental Microbiology. 2000; 69(10): 5974 - 5982.

27. Van Hamme J, Ajay S, et al. Recent advances in petroleum Microbiology. Microbiol Mol Biol Rev. 2000; 67(4): 503-549.

28. Torres R. El papel de los microorganismos en la biodegradación de compuestos tóxicos. Ecosistemas. 2003 http://www.aeet.org/ecosistemas/032/informe1.html.

29. Flavio B, Leonardo F, Zachia M. Production of biosurfactant by hydrocarbon degrading Rhodococcus ruber and Rhodococcus erythropolis. Revista de Microbiología. 1999; 30 (3).

30. Eriksson M, Sodersten E, Zhongtang Y, Dalhammar G, Mohn W. Degradation of polycyclic aromatic hydrocarbons at low temperature under aerobic and nitrate-reducing. Appl Environ Microbiol. 2003; 69(1): 275-284

31. Carla A, Nicholson M, Fathepure B. Biodegradation of benzene by halophilic and halotolerant bacteria under aerobic conditions. Applied and Environmental Microbiology. 2004; 70(2): 1222-1225.

32. Hideki K, Katsuyuki U, Hisako H, Horikoshi K. Novel toluene elimination system in a toluene-tolerant microorganism. Journal of Bacteriology. 2000; 182: 6451-6455.
33. Kästner M, Breuer M, Mahro B. Impact of inoculation protocols, salinity, and $\mathrm{pH}$ on the degradation of Polycyclic Aromatic Hydrocarbons (PAHs) and survival of PAHdegrading Bacteria introduced into soil. Applied and Environmental Microbiology. 1998; 64(1): 359-362.

34. Sudarat B, Britz M, Grant S. Degradation and mineralization of high-molecular-weight polycyclic aromatic hydrocarbons by defined fungal-bacterial cocultures. Applied and Environmental Microbiology.2000; 66(3): 1007-1019.

35. Nelson K, Weinel C, Paulsen I, Dodson R, Hilbert H, Martins dos Santos V, Fouts D, Gill S, Pop M, Holmes M, et al. Complete genome sequence and comparative analysis of the metabolically versatile Pseudomonas putida KT2440. Environmental Microbiology. 2002; 4: 799-813.

36. Rockne K, Chee-Sanford J, Sanford R, Brian P, James T, Staleyand S. Anaerobic naphthalene degradation by microbial pure cultures under nitrate-reducing conditions. Applied and Environmental Microbiology . 2000; 66: (4)1595-1601.

37. Fan C, Qing X, Kwang J. Aerobic Denitrification of Pseudomona aeruginosa monitored by online NAD(P)H Fluorescence. Applied And Enviromental Microbiology. 2003; 69 (11): 6715-6722.

38. Gesche B, Andreas F, Witzel K. Development of PCR primer system amplification of nitrite reductase genes (NirK anç nirS) to detect denitrifying bacteria in environmental samples. 1998.

39. Demaneche S, Kay E, Gourbiere F, Simonet P. Natural transformation of Pseudomonas fluorescens and Agrobacterium tumefaciens in soil. Applied and Environmental Microbiology. 2001; 67 (6): 2617-2621.

40. Svenja R, Alexander N, Ulf S, Peter K. Differential detection of key enzymes of polyaromatic-hydrocarbon-degrading bacteria using PCR and gene probes. Microbiology. 1999; 145: 1731-1741.

41. Díaz M, Grigson S, Burgess J. Uso de un consorcio bacteriano extremo-halotolerante para la biodegradación de crudo en ambientes salinos. Revista Colombiana de Biotecnología. 2002; 4(1): 36-42.

42. Floriane S, Marchal R, Serge C, Christelle V, Lebeault J, Vandecasteele J. A Mycobacterium strain with extended capacities for degradation of gasoline hydrocarbons. Microbiology. 2000; 66( 6): 2392-2399.

43. De Lorenzo V. Biorremediación: De la placa de petri al medio ambiente. Centro Nacional de Biotecnología.2002. En: www.bioprest.net.

44. Margarida M, Pozzi E, Campos J, Cleto R, Mattos E. Estimate of denitrifying microbiota in tertiary sewage treatment and kinetics of the denitrification process using different sources of carbon. Brazilian journal of Microbiology. 2003; 34: (2).

45. Christopher W. Kaplan R, Christopher L. Bacterial succession in a petroleum land treatment unit. Appl Environ Microbiol. 2004; 70(3): 1777-1786.

46. Nannipieri P, Ascher J, Ceccherini M, Landi L, Pietramellara M, Renella G. Microbial diversity and soil functions. 2001. European Journal Soil Science. 2001;54(4): 655-670.

47. De Boer W, Verheggen P, Gunnewick K, Kowalchuk G, and Veen J. Microbial Community Composition Affects Soil Fungistasis. Applied and Environmental Microbiology. 2003; 69(2): 835-844.

48. Jizhong Z, Beicheng X, David T, Wu L, Terry M, Robert Neill, Anthony P, and James M. Spatial and resource Factors Influencing High Microbial Diversity in soil. Environmental Sciences Division. Center for Microbial Ecology, Michigan State University, Michigan. 2001.

49. López J, García O, Grima J, Ballesteros J, Pérez. Técnicas de biorrecuperación in situ en acuíferos contaminados por metales pesados. 2000.

50. Sanjeet M, Jeevan J, Ramesh C, Banwarj L. Evaluation of Inoculum Addition To Stimulate in situ Bioremediation of Oily-Sludge- Contaminated Soil. Applied And Enviromental Microbiology. 2001; 67(4):1675-1681. 